en globalen Organisation, in der Demokratien die Zusammenarbeit bei gemeinsamen Herausforderungen koordinieren, die außenpolitische Debatte in den USA weiterhin beschäftigen. Denn er hat lediglich einen Vorschlag aufgenommen, der vor einiger Zeit unter der Bezeichnung „Concert of Democracies“ auch von liberalen Vordenkern in diese Debatte eingebracht wurde. ${ }^{39}$

Eine solche Organisation soll die Kooperation zwischen liberalen Demokratien stärken und einen institutionellen Rahmen für die Zusammenarbeit bei der Bewältigung gemeinsamer globaler Probleme bereitstellen. Insbesondere gilt dies für „harte“ sicherheitspolitische Probleme, bei denen die Vereinten Nationen diese Rolle aufgrund der Entscheidungsverfahren im UNSicherheitsrat nicht oder nur unzureichend ausüben können. So unterschiedlich die Vorschläge in ihrer Reichweite auch sind, so laufen sie doch auf den Abschied von einem auf die Vereinten Nationen fokussierten globalen Multilateralismus und den Aufbau eines konkurrierenden Legitimationssystems für den Einsatz militärischer Macht hinaus. Unter Neokonservativen ist die Idee eines „Bundes der Demokratien“ in die ReIdeologisierung internationaler Politik eingebettet. Diese ideologische Verortung des Zusammenschlusses der Demokratien

39 Hierzu siehe Peter Rudolf, Ein „Bund der Demokratien“: Amerikas neuer globaler Multilateralismus?, Berlin: Stiftung Wissenschaft und Politik, April 2008 (SWP-Aktuell 28). spiegelt sich deutlich in der Programmatik von John McCain wider, der ein neues „organisierendes Prinzip“ der amerikanischen Außenpolitik im Sinn hat, das über das Paradigma des „Kriegs gegen den Terror“ hinausgeht. Die Auseinandersetzung mit „internationalen Terroristen“ wird in einen breiteren, älteren ideologischen Konflikt eingereiht: in den weltweiten Kampf zwischen „Freiheit und Despotismus“. In diesen Horizont wird der Konflikt mit einem autoritären „revanchistischen“ Russland eingeordnet, das McCain als Präsident aus der G 8 ausschließen (und dafür Indien und Brasilien aufnehmen) will, vor allem aber der Konflikt mit dem radikalen Islam, wobei hier die iranischen Mullahs, al-Qaida und die Hizbollah in einem Atemzug genannt werden.

In dieses neue organisierende Prinzip fügt sich auch der „weltweite Bund der Demokratien“ ein. Er würde den „Kern einer auf Freiheit gegründeten internationalen Friedensordnung“ bilden, erklärtermaßen nicht als Ersatz, aber als Ergänzung der Vereinten Nationen. Das Projekt eines „Bundes der Demokratien" offeriert Senator McCain den amerikanischen Verbündeten als neuen "grand bargain“: als amerikanische Bereitschaft, ihre Positionen zu beachten und zu respektieren, im Austausch gegen deren Bereitschaft, bei der gemeinsamen Verteidigung von Freiheit, Demokratie und wirtschaftlicher Wohlfahrt mitzuwirken - auch mit militärischen Mitteln.

\title{
Die transatlantischen Beziehungen nach der Ära Bush
}

\author{
Karsten D. Voigt*
}

\begin{abstract}
Whenever a new US President takes power, there are hopes for a fresh start in American foreign and security policy. The administration due to take office in 2009 will be no exception. The election of a new President and a new Congress could be an opportunity for closer transatlantic cooperation on major issues. However, hopes for cooperation without disagreements are unrealistic. The US will not give up the option of acting unilaterally when important US interests are at stake, and Europe still lacks the will and the capability to assume a much greater share of the burden of maintaining international security and stability. Yet the new US administration can be expected to become more pragmatic and restrained in rhetoric and style in order to secure the Europeans' support for US policy in the Middle East, Iran, Afghanistan and other trouble spots. Washington may even be willing to truly consult its allies before acting. The new administration will engage more in non-traditional foreign policy topics, such as climate change and energy security, which could bring US policies more in line with European priorities. In its own interest, the EU should respond positively to American overtures, because it only stands a chance of becoming a strong and relevant international actor in partnership with the United States.
\end{abstract}

Key Words: US presidential election, transatlantic relations, security policy, climate protection, Transatlantic Economic Council, globalization

US-Präsidentschaftswahl, transatlantische Beziehungen, Sicherheitspolitik, Klimaschutz, transatlantischer Wirtschaftsrat, Globalisierung.

\section{Die Präsidentschaftswahl 2008: Neuanfang in den transatlantischen Beziehungen?}

$\mathrm{W}$

enn der 44. Präsident der Vereinigten Staaten im Januar 2009 sein Amt antritt, begleiten ihn nicht nur im eigenen Land, sondern auch bei den Verbündeten zahlreiche Hoffnungen auf einen Neuanfang. Ein neuer Mann oder eine neue Frau an der Spitze des mächtigsten
Landes der Welt ist für die Menschen auf allen Kontinenten relevant, ob sie nun in Chicago, Leipzig oder einem iranischen Dorf wohnen. In einer Erhebung von Harris Interactive vom November $2007^{1}$ sagten beispielsweise 37 Prozent der befragten

\footnotetext{
Karsten D. Voigt ist seit 1999 Koordinator für die deutsch-amerikanische Zusammenarbeit im Auswärtigen Amt, Berlin. Dieser Artikel gibt seine persönliche Meinung wieder.

1 Harris Interactive: The Talk of Paris. A Harris Interactive Survey of The Talk of Paris, France 24 and the International Herald Tribune. Dezember 2007, S. 8.
} 
Deutschen, sie seien „sehr“ oder „äußerst interessiert“ an den US-Präsidentschaftswahlen. Nur 19 Prozent zeigten kein Interesse an diesem Thema. Vor allem in diesem Wahljahr, in dem weder der bisherige Präsident noch der Vizepräsident antreten, ist die Sache unvorhersehbar und damit besonders spannend. Verschiedene Kandidaten auf der demokratischen, aber auch auf der republikanischen Seite präsentieren sich dem Wahlvolk als Garanten des Wandels und der Abkehr von der in weiten Teilen der USA als zynisch wahrgenommenen Washingtoner Politik. Laut Umfragen im Januar 2008 sind ca. 60 Prozent der Amerikaner mit der Politik des bisherigen Präsidenten unzufrieden $^{2}$ und fast 70 Prozent glauben, das Land sei außenpolitisch auf dem falschen Weg. ${ }^{3}$

Noch niederschmetternder fällt das Urteil über den Präsidenten und die amerikanische Außenpolitik in vielen Teilen der Welt aus, vor allem in der arabischen Welt, aber auch bei den Partnern und Verbündeten in Europa. Der ,German Marshall Fund of the United States' (GMF) veröffentlichte im September 2007 Umfrageergebnisse (,Transatlantic Trends: Key Findings 2007'4) aus zwölf europäischen Ländern (einschließlich der Türkei) und den USA, nach denen lediglich 36 Prozent der befragten Europäer eine Führungsrolle der USA in der Welt für wünschenswert erachten. Im Jahr 2002, also vor dem unpopulären Irakkrieg, lag diese Zahl noch bei 64 Prozent. Besonders stark war der Absturz der Umfragewerte in diesem Zeitraum in Deutschland von 68 auf 38 Prozent. Die Zustimmung der Europäer zu dem von Anfang an nicht sonderlich beliebten Präsident Bush sank derweil von 38 auf 17 Prozent. Während sich die Beziehungen zwischen den Regierungschefs in Berlin und Washington und auch Paris und Washington wieder deutlich verbessert haben, bleibt die Bevölkerung in Europa der US-Regierung gegenüber sehr kritisch eingestellt.

Die schlechte Bewertung der amerikanischen Außenpolitik und des verantwortlichen Führungspersonals ist sowohl für die USA als auch für die europäischen Alliierten, die mit Amerika in einer Werte- und Interessengemeinschaft verbunden sind, negativ. Kein Wunder also, dass nun viele Hoffnungen auf der nächsten Administration ruhen, die Führungsrolle der USA wieder glaubwürdig und effektiv zu gestalten, Amerika als freiheits- und wohlstandsverheißende Idee neu zu begründen und die Partnerstaaten bei der Lösung internationaler Probleme zu konsultieren.

Ist es jedoch realistisch anzunehmen, dass sich das Einverständnis, das zwischen Amerikanern und Europäern während des Kalten Krieges bestand, nach den US-Präsidentschaftswahlen schnell wieder herstellen lässt? Die Europäer sind da skeptisch. In den bereits zitierten Umfragen im Auftrag des German Marshall Fund äußerten sich nur 37 Prozent der befragten Europäer optimistisch, dass sich die beiderseitigen Beziehungen nach den Wahlen von 2008 verbessern; 46 Prozent glauben, dass die transatlantischen Beziehungen unverändert bleiben werden, in Deutschland sogar 54 Prozent und in Spanien 52 Prozent der

2 Pew Research Center for the People \& the Press. Survey conducted by Princeton Survey Research Associates International. Jan. 9-13, 2008.

3 Scott Bittle, Jonathan Rochkind u.a.: Loss of Faith. Public's Belief in Effective Solutions Eroding, in: Public Agenda: Confidence in U.S. Foreign Policy Index, unter: http://www. publicagenda.org/foreignpolicy/pdfs/ foreign_policy_index_fall07.pdf\#page $=14$. Herbst 2007.

4 Ebenda.
Befragten. So wichtig ein neuer Präsident oder eine neue Präsidentin sein mag - vermutlich haben die Menschen mit ihrer instinktiven Skepsis recht. Zu tiefgreifend war das Unverständnis in weiten Teilen der europäischen Bevölkerung über den Irakkrieg, über die Klimapolitik der gegenwärtigen Administration und über einen als dominant und missionarisch empfundenen politischen Stil. Auf amerikanischer Seite wiederum machte sich Enttäuschung über die europäischen Verbündeten breit, die man als wenig relevant für die Lösung konkreter sicherheitspolitischer Probleme wahrnahm.

\section{Was tun gegen neue Gefahren?}

Bei aller guten Zusammenarbeit werden also viele der in den letzten Jahren zwischen den transatlantischen Partnern aufgebrochenen Differenzen bleiben, denn sie haben weniger mit Präsident Bush, als vielmehr mit den geopolitischen Veränderungen nach der Zeitenwende von 1989 und den Terroranschlägen vom 11. September 2001, mit den Unterschieden in der Machtposition und der Rolle im internationalen System sowie den Differenzen in der politischen Kultur zu tun. Im Gegensatz zum Kalten Krieg gestaltet sich die Bedrohungslage für die westlichen Staaten heute unübersichtlich und unvorhersehbar. Das Terrornetzwerk al-Quaida ist nicht territorial bestimmbar. Wir wissen noch zu wenig über diesen in unabhängigen Zellen vorgehenden Feind, der seine Anschläge mit großer Skrupellosigkeit verübt und sich militärischer Eindämmung geschmeidig entzieht. Terroristen machen sich sogenannte „failed states“ wie Afghanistan unter den Taliban oder Somalia zunutze. Sie operieren zudem vom Staatsgebiet mit den USA verbündeter Länder wie Pakistan und sogar als terroristische Zellen aus der Mitte europäischer Gesellschaften heraus.

Die letzten Jahrzehnte haben zudem neu aufstrebende Mächte wie China und Indien hervorgebracht. Der Wiederaufstieg eines machtbewussten und mit gewaltigen Energievorräten gesegneten Russlands sowie die Bedrohung durch aggressiv auftretende, autoritär geführte Staaten, die Atomwaffen besitzen oder unter dem Verdacht stehen, eine nukleare Bewaffnung anzustreben, sind weitere Beispiele für Veränderungen im internationalen System. Die westlichen Demokratien suchen immer noch nach den wirksamsten Mitteln, den neuen Bedrohungen zu begegnen.

Demokratieförderung darf sich nicht nur auf die Abhaltung von Wahlen beschränken. In Ländern, in denen der liberale Individualismus keine Tradition hat, wählen die Menschen insbesondere in den ersten Jahren nach dem Ende autoritärer Regime häufig gemäß kollektiver Identitäten, also entlang ihrer religiösen und ethnischen Orientierung. In diesen Gesellschaften sind zusätzlich zu Wahlen nach dem Prinzip „eine Stimme für jeden Staatsbürger“, Regeln für den Ausgleich der Interessen zwischen einzelnen Gruppen unabdingbar. Wo diese aber fehlen und Rechtsstaatlichkeit, gute Regierungsführung, ein Minimum an sozialer Gerechtigkeit und öffentlicher Sicherheit nicht gewährleistet sind, wird man mit noch so gut gemeinten Demokratisierungsbestrebungen nur die Demokratie selbst diskreditieren. Die westliche Politik im Nahen Osten und in Afrika hat in den letzten Jahrzehnten insofern zwar verbündete Regie- 
rungen hervorgebracht, aber zu wenig für die Verbesserung der Lebensverhältnisse der Menschen bewirkt.

Die nach 1989 gewachsene Überzeugung, früher oder später werde die ganze Welt zwangsläufig auf den westlich liberalen Zug aufspringen, ${ }^{5}$ ist jedenfalls dem beunruhigenden Gefühl gewichen, dass die westlichen Werte von liberaler Demokratie und Marktwirtschaft nicht überall auf dem Vormarsch sind und sich in einer sich wieder verstärkenden Konkurrenz mit autoritären Gesellschaftsentwürfen durchsetzen müssen.

\section{Militärische Übermacht versus "Soft Power“}

Amerikaner und Europäer gehen bei der Bewältigung neuer Bedrohungslagen aufgrund ihrer unterschiedlichen Rolle und Machtmittel von unterschiedlichen Voraussetzungen aus . Die USA mit ihrer weltweiten Machtprojektion und dem Bewusstsein ihrer Verantwortung für die Stabilität der gesamten Welt sind nach wie vor bereit, international beträchtliche Mittel in die Vertretung ihrer Interessen zu investieren. Sie wenden fast vier Prozent ihres Bruttoinlandprodukts für Verteidigung auf und sind selbstverständlich bereit, für die Wahrung ihrer Sicherheit und ihrer Interessen militärische Mittel einzusetzen.

Für die europäischen ständigen Mitglieder des UN-Sicherheitsrats, Frankreich und das Vereinigte Königreich, mag dies noch mit Abstrichen gelten, für Deutschland allerdings nicht. Zwar hat sich Deutschland in einem Lernprozess im Lauf der 1990er Jahre den neuen internationalen Herausforderungen gestellt und beteiligt sich mit derzeit rund 6.500 Soldaten an Einsätzen im Ausland, aber die Bevölkerung lehnt sämtliche Einsätze, einschließlich des Afghanistan-Engagements, mehrheitlich ab, auch wenn sie große Mehrheiten bei den jeweiligen Mandatsverlängerungen im Deutschen Bundestag erhalten. Die politische Elite bemüht sich, die Akzeptanz der Auslandseinsätze zu verbessern, indem sie deren demokratie- und entwicklungsfördernde Ziele betont. Dass die Bundeswehr, wenn nötig aber auch zu Kampfeinsätzen bereit sein muss, wird in der deutschen Öffentlichkeit immer noch nicht offen genug gesagt und gegenüber der weiterhin skeptischen Bevölkerung nicht überzeugend genug begründet.

Wie andere europäische Armeen auch, entwickelt die Bundeswehr schrittweise Fähigkeiten, um ihrem neuen Aufgabenspektrum gerecht zu werden. Den Amerikanern gehen diese Prozesse in Europa nicht schnell genug. Im Januar 2008 kritisierte der amerikanische Verteidigungsminister Gates die NATO-Verbündeten, sie seien zu schlecht ausgebildet, um Aufständische in Afghanistan wirkungsvoll zu bekämpfen. ${ }^{6}$ Gleichzeitig kündigte das Pentagon an, zusätzlich 3.200 Soldaten nach Afghanistan zu entsenden. Aus Washingtoner Sicht wird die EU die Vereinigten Staaten bei der Wahrung der internationalen Stabilität daher zwar weiterhin unterstützen, auf absehbare Zeit aber nicht ersetzen können. Allerdings kann Europa seine Erfahrungen mit dem zivilen Aufbau von Gesellschaften, mit der

\footnotetext{
5 Fukuyama, Francis. “The End of History?” The National Interest. Summer 1989, S. 3-18.

6 Peter Spiegel: Gates faults NATO force in southern Afghanistan. In: Los Angeles Times January 16, 2008, unter: http://www.latimes.com/news/nationworld/world/la-fg-usaf-
} ghan16jan16,0,1957179.story.
Integration verschiedener politischer Mittel für die Stabilisierung von Krisenregionen und schließlich auch seine Einsicht in die Langwierigkeit politischer Veränderungen einbringen.

Die missionarische Ausrichtung der US-Außenpolitik während der ersten Amtsjahre von Präsident Bush hat sich in der zweiten Amtsperiode zugunsten einer stärker realpolitischen Orientierung abgeschwächt. Es wuchs die Einsicht, dass man mit Streitkräften zwar Siege erringen, aber nicht langfristig Verbündete gewinnen und Interessen verteidigen kann. US-Verteidigungsminister Gates rief in einer Rede an der Kansas State University vom 26. November 2007 die USA dazu auf, neben der militärischen ,hard power' künftig mehr ,soft power' einzusetzen und diese mit den militärischen Mitteln zu verbinden. ${ }^{7}$ In den asymmetrischen Konflikten der letzten Jahre im Irak, in Afghanistan, auf dem Balkan und in Somalia habe sich gezeigt, dass militärische Erfolge allein nicht ausreichen, um Sicherheit zu produzieren. Es müssten auch die zivilen Instrumente der nationalen Sicherheit wie Diplomatie, Wiederaufbau- und Entwicklungszusammenarbeit und strategische Kommunikation gestärkt werden. Zudem müsse man andere Staaten viel mehr dabei unterstützen, sich selbst zu regieren.

Dies ist eigentlich eine alte Weisheit. Auch aus den Westdeutschen wurden nach 1945 nicht über Nacht pro-amerikanische Musterdemokraten. Vielmehr wurden die Herzen der Deutschen über die Jahre mit Care-Paketen, dem Marshall-Plan, dem Aufbau eines funktionierenden neuen Staates, dem gemeinsamen Engagement gegen die sowjetische Bedrohung und mit Hilfe der amerikanischen Musik- und Filmindustrie gewonnen. Es sei in diesem Kontext auch daran erinnert, dass das sowjetische Imperium zwar durch die militärische Macht der NATO an der Expansion gehindert, letztlich aber nicht militärisch besiegt wurde. Es zerfiel, weil es sich auf Dauer im Wettbewerb mit dem westlichen Wirtschafts- und Gesellschaftsmodell als dysfunktional und unattraktiv erwies.

\section{Was kann man von der neuen Administration erwarten?}

Niemand kann vorhersehen, wer 2009 in die Pennsylvania Avenue 1600 einziehen wird. Vermutungen über politische Trends der neuen Administration sind jedoch legitim und werden in den Medien und in der Fachwelt bereits breit diskutiert. Das amerikanische Volk ist zwar weiterhin bereit, die internationale Führungsrolle der USA und das mit Abstand größte Militärbudget der Welt zu finanzieren. Es ist jedoch aufgrund der Erfahrungen der letzten Jahre sehr viel misstrauischer gegenüber den jeweiligen Begründungen von Militäreinsätzen geworden. Und auch die Medien stellen - anders als in der patriotischen Stimmung nach dem 11. September 2001 - wesentlich kritischere Fragen an die Regierenden.

Vor allem wäre ein US-Militärschlag gegen Iran momentan weder der amerikanischen Bevölkerung noch den Verbündeten zu vermitteln. Die Veröffentlichung des ,National Intelligence

\footnotetext{
7 Robert M. Gates, speech delivered at Kansas State University November 26, 2007, unter: http://www.defenselink.mil/speeches/speech.aspx?speechid=1199.
} 
Estimate' vom November $2007^{8}$ laut dem Iran im Herbst 2003 sein Nuklearwaffenprogramm stoppte, hat die Wahrscheinlichkeit eines Angriffs auf iranische Nuklearanlagen und andere Ziele in der nächsten Zukunft weiter reduziert. Allerdings will zum jetzigen Zeitpunkt keiner der Bewerber um die Präsidentschaftsnominierung eine militärische Option ausschließen.

Allgemein ist von der neuen Administration eine zurückhaltendere politische Rhetorik und angesichts der Erfahrungen im Irak mehr Vorsicht bei neuen militärischen Engagements zu erwarten. Die laufenden Einsätze werden aber sicherlich konsequent fortgeführt werden. Was auch immer die Kandidaten im Vorwahlkampf sagen, kein demokratischer oder republikanischer Oberkommandierender wird die US-Truppen abrupt aus dem Irak abziehen. Dies würde nicht nur die Stabilisierungserfolge der letzten Monate zunichte machen, sondern könnte auch zu weiteren Einmischungen durch die Nachbarstaaten und zum Auseinanderbrechen des Landes führen. Man wird also versuchen, je nach Lage und Stabilitätsfortschritt die Truppen schrittweise aus dem Irak abzuziehen und die Verantwortung an die irakischen Behörden zu übergeben. In dem Maße, in dem dies gelingt, werden sich die USA stärker auf den eigentlichen Anti-Terror-Kampf in Afghanistan und auf die Situation in Pakistan konzentrieren. Gegenüber den europäischen Verbündeten werden dabei die Forderungen nach Entlastung und Unterstützung der USA zunehmen.

Dies gilt auch für die angestrebte Friedenslösung im Nahen Osten, die auch unter der neuen Administration ein sehr wichtiges außenpolitisches Ziel der USA bleiben wird. Selbst wenn, wie bei der Nahost-Konferenz in Annapolis am 27. November 2007 vorgezeichnet, die Kernfragen (Grenzen zwischen Israel und einem palästinensischen Staat, die Kontrolle über Jerusalem und die Rückkehr bzw. Entschädigung palästinensischer Flüchtlinge) bis zum Ende der Amtszeit George W. Bushs in Verhandlungen geregelt werden könnten, müssten diese Vereinbarungen in der Amtszeit der neuen US-Regierung verwirklicht werden. Der Prozess selber wird dabei noch mehrfach ins Stocken geraten oder vom Scheitern bedroht sein. Damit er zu einem positiven Ergebnis geführt werden kann, werden von den Konfliktparteien viel Kompromissbereitschaft und von den USA und Europa mühevolle Überzeugungsarbeit nötig sein. Über die Erfolgsaussichten ist allenfalls ein sehr vorsichtiger Optimismus angebracht.

Vor allem falls ein Demokrat oder eine Demokratin im Januar 2009 ins Weiße Haus einzieht, könnte die amerikanische Außenpolitik stärker als in den vergangenen Jahren bereit sein, multilaterale Institutionen ernster zu nehmen als bisher und auch die Position befreundeter Staaten stärker bei der eigenen Entscheidungsfindung zu berücksichtigen. Aber auch ein republikanischer Präsident wird voraussichtlich - insbesondere zu Beginn seiner Amtszeit - mit neuen kooperativen Initiativen auf die Europäer zugehen. Die Europäer sollten auf entsprechende Signale aus Washington möglichst kooperativ reagieren. Dass die neue US-Regierung den Multilateralismus in gleicher Weise wie beispielsweise Deutschland als Wert an sich und das Völkerrecht als nationale Entscheidungen von

8 National Intelligence Council: Iran: Nuclear Intentions and Capabilities. November 2007, unter: http://www.dni.gov/press_releases/20071203_release.pdf.
US-Kongress und Präsident überlagernde Autorität anerkennt, ist aber ausgeschlossen. Dem widerspräche nicht nur die unterschiedliche verfassungspolitische Tradition, sondern auch der Weltmachtstatus und die politische Kultur der Vereinigten Staaten.

Letztlich wird kein US-Präsident die Anwendung militärischer Gewalt und dabei auch unilaterales Handeln ohne Verbündete ausschließen, wenn es um die Verteidigung wichtiger amerikanischer Sicherheitsinteressen geht. Wörtlich schreibt zum Beispiel der in den europäischen Medien als Erneuerer gefeierte Senator Obama in seinem Beitrag, Renewing American Leadership' in der Zeitschrift Foreign Affairs vom Juli/August 2007: ,I will not hesitate to use force, unilaterally if necessary, to protect the American people or our vital interests whenever we are attacked or imminently threatened "9. Alle anderen Bewerber sehen dies genauso. Transatlantische Meinungsunterschiede sind weniger als viele Deutsche es heute glauben in Personen als in der unterschiedlichen geostrategischen Situation und in unterschiedlichen politischen Kulturen beider Seiten des Atlantiks begründet.

\section{Kooperation und Lastenteilung}

Dort, wo die USA bereit sind, die Verbündeten in die Lösung von Konflikten einzubeziehen, werden sie auf eine gerechtere Lastenverteilung pochen und Deutschland und die EU mit neuen Forderungen nach der Übernahme militärischer und ziviler Aufgaben in Krisengebieten konfrontieren. Aus den außenpolitischen Strategiepapieren der amerikanischen Präsidentschaftsbewerber ist dies deutlich herauszulesen. So spricht Senator Obama in seinem bereits erwähnten Beitrag für Foreign Affairs davon, dass er in Afghanistan die Truppen verstärken und zugleich daran arbeiten wolle, die „Beschränkungen, die einige NATO-Verbündeten ihren Truppen auferlegen, zu beseitigen“. In ähnlicher Weise äußert sich zum Beispiel auch Senator McCain (Foreign Affairs vom November/Dezember 2007) ${ }^{10}$.

Diese kaum verhüllten Hinweise richten sich auch an Deutschland, das seinen ISAF-Einsatz gemäß Bundestagsmandat auf den bisher relativ friedlichen Norden Afghanistans begrenzt. Verteidigungsminister Gates äußerte im Dezember 2007 vor dem Streitkräfteausschuss des Repräsentantenhauses Kritik am Einsatz der europäischen Verbündeten am Hindukusch. Dies gelte vor allem für die Ausbildung afghanischer Sicherheitskräfte. Der amerikanische Druck auf die EU und auf Deutschland, mehr in Afghanistan und für die gemeinsame Sicherheit zu leisten, wird sich noch verstärken. Die Lösung von Konflikten in Europa, vor allem die Sicherung der Stabilität im Kosovo und andernorts auf dem Balkan, werden die Amerikaner zunehmend als europäische Aufgabe verstehen. Nach der (zu erwartenden) Unabhängigkeitserklärung des Kosovo von Serbien sehen die USA die Lösung der Folgeprobleme weitgehend als Aufgabe der Europäer an. Das Engagement der Europäischen

9 Barack Obama: Renewing American Leadership, in: Foreign Affairs Juli/August 2007, unter: http://www.foreignaffairs.org/ 20070701 faessay86401-p10/barack-obama/renewing-american-leadership.html.

10 John McCain: An Enduring Peace Built on Freedom, in: Foreign Affairs November/ Dezember 2007, unter: http://www.foreignaffairs.org/20071101faessay86602/john-mccain/an-enduring-peace-built-on-freedom.html. 
Sicherheits- und Verteidigungspolitik wird langfristig und die Perspektive der Aufnahme der betreffenden Staaten in die EU wird glaubwürdig und konkret sein müssen, um zu verhindern, dass nationale und ethnische Konflikte wieder aufbrechen.

Angesichts der erheblichen Skepsis in der öffentlichen Meinung zu den Auslandseinsätzen der Bundeswehr wird es für die deutsche Regierung und den Bundestag noch schwieriger, aber auch noch wichtiger werden, für die richtigen außen- und sicherheitspolitischen Entscheidungen zu werben. Wir werden nicht überall in der Welt eingreifen können, wo es zu Konflikten kommt. Aber prinzipiell werden die Anforderungen an Deutschland wachsen. Diese Anforderungen mit dem Hinweis auf die deutsche Geschichte zurückzuweisen, wird moralisch immer unglaubwürdiger werden und politisch immer weniger begründbar sein. Man kann aber jetzt schon sicher sein, dass die Linkspartei das Unbehagen der Bevölkerung über die Auslandseinsätze bei der Bundestagswahl 2009 für sich instrumentalisieren wird. Wie auch bei Entscheidungen über die Einführung des Euro und Änderungen der EU-Verträge verbinden sich auch bei diesem Thema bei der Linkspartei ein rhetorischer Internationalismus mit einem faktischen Nationalismus.

In allen internationalen Konflikten, die für Europa und die USA eine Rolle spielen, ist die Position Russlands ein wesentlicher Faktor. Eine trotz aller Meinungsunterschiede konstruktive Zusammenarbeit mit Moskau erleichtert es, im Atomstreit mit Iran, in der Kosovo-Frage, bei der künftigen außenpolitischen Orientierung Serbiens oder bei Konflikten im postsowjetischen Raum tragfähige Lösungen zu erzielen. Die Haltung der US-Regierung und vor allem auch des US-Kongresses gegenüber Russland ist zunehmend kritisch geworden. Auch von Politkern der Demokraten wird Russland immer mehr als Gegenspieler und als Bedrohung für amerikanische Interessen wahrgenommen. Wir Deutsche empfinden die autoritären Tendenzen in Russland ebenfalls als Widerspruch zu unseren eigenen Werten. Trotzdem bleibt es unser Interesse, eine enge politische, wirtschaftliche und kulturelle Zusammenarbeit Russlands mit dem Westen insgesamt und insbesondere mit der Europäischen Union zu fördern. Vor allem ist ein neuer Dialog mit Russland über Abrüstung, Rüstungskontrolle und Nichtverbreitung dringend notwendig.

\section{Neue Kooperationsfelder}

Die militärische und zivile Krisenbewältigung wird in den nächsten Jahren ein wichtiges Thema der transatlantischen $\mathrm{Zu}$ sammenarbeit bleiben. Aber auch bei sogenannten, soft issues' wie dem internationalen Klimaschutz, der Energiesicherheit, der Wasserversorgung, der Entwicklung, der Migration und der Globalisierung der Wirtschaft werden wir mit der neuen amerikanischen Regierung kooperieren und gemeinsam Fortschritte erzielen können.

Nehmen wir z.B. den Klimaschutz: Die von George W. Bush geführte Regierung hat sich damit schwer getan. Sie lehnt, ebenso wie der gesamte US-Senat, das Kyoto-Protokoll zur Begrenzung von Treibhausgasen ab, weil es ihrer Auffassung nach das Wirtschaftswachstum begrenzt und große Schwellenländer wie Indien und China, die einen immer größeren Anteil am Ausstoß klimaschädlicher Emissionen haben, nicht in die Pflicht nimmt. Mittlerweile hat sich jedoch auch die Diskussion in den USA verändert. Zum einen weist der Stand der wissenschaftlichen Erforschung des Klimawandels mittlerweile so eindeutig auf eine von Menschen verursachte Entwicklung hin, ${ }^{11}$ dass auch die Republikaner den Klimawandel als Faktum anerkennen. Die Maßnahmen, mit denen das Weltklima am wirkungsvollsten geschützt und die Wirtschaft zugleich vor übermäßiger Regulierung bewahrt werden kann, bleiben aber strittig. Die US-Administration setzt weiterhin auf freiwillige Lösungen und technologische Fortschritte, während die Europäer für eine verbindliche Verminderung von Treibhausemissionen eintreten.

Bei der Klimakonferenz in Bali im Dezember 2007 kam es zu einer Einigung über ein Verhandlungsmandat für ein neues Vertragswerk, das die internationale Klimapolitik nach dem Auslaufen des Kyoto-Protokolls 2012 bestimmen soll. Bindende Ziele zur Emissionsverringerung konnten in Bali zwar nicht vereinbart werden, aber das Mandat ermöglicht es, im nun folgenden Prozess Minderungsziele auch für die USA und für die Schwellenländer zu verhandeln. Weite Teile der Bevölkerung der USA wären allerdings zu weiteren Schritten bereit. Nicht zuletzt die verheerenden Auswirkungen des Hurricane Katrina im August 2005 haben der amerikanischen Öffentlichkeit einen Vorgeschmack auf zu erwartende Umweltkatastrophen durch den Klimawandel gegeben. Es ist in großstädtischen Eliten modern geworden, sich umwelt- und klimabewusst zu geben. Hollywood erweist sich mit den Hybridautos seiner Filmstars einmal mehr als Trendsetter. Selbst evangelikale Christen haben das Thema unter dem Aspekt der Bewahrung der Schöpfung für sich entdeckt.

Eine Reihe von Bundesstaaten haben eigene Gesetze und Richtlinien zur Begrenzung von klimaschädlichen Emissionen und zur Förderung erneuerbarer Energien verabschiedet. Darunter sind vor allem das progressive Kalifornien unter Gouverneur Schwarzenegger, aber auch andere große Staaten wie Florida und Colorado zu nennen. Kalifornien hat sogar gemeinsam mit fünfzehn weiteren US-Bundesstaaten und fünf Umweltorganisationen die Bundesregierung in Washington verklagt, um schärfere Grenzwerte für die Luftreinhaltung durchzusetzen als vom entsprechenden Bundesgesetz und der Umweltbehörde EPA vorgesehen. Auch eine Reihe von US-Städten haben sich zu einem Klimabündnis zusammengeschlossen, und eine ,ApolloAlliance“ aus Gewerkschaften, Unternehmen, Umweltgruppen und Kommunalpolitikern wirbt für erneuerbare Energien und Energieeffizienz.

Der Boden ist also durchaus fruchtbar für eine verstärkte $\mathrm{Zu}$ sammenarbeit zwischen den USA und Europa nach den Präsidentschaftswahlen vom kommenden November. Bundesaußenminister Steinmeier hat bereits im August 2007 Kalifornien besucht, um Möglichkeiten für eine engere transatlantische $\mathrm{Zu}$ sammenarbeit in Energie- und Klimafragen auszuloten. Auch in den USA wird sich der Druck durch die öffentliche Meinung, die schwindenden Ölreserven, steigende Ölpreise und durch

11 siehe Ergebnisse des Intergovernmental Panel on Climate Change der Vereinten Nationen: AR4 Synthesis Report. November 2007, unter: http://www.ipcc.ch/ipccreports/ar4syr.htm. 
die wirtschaftlichen Folgen des Klimawandels verstärken. Hinzu kommen Sorgen um die Energiesicherheit, denn 60 Prozent der verbleibenden Ölreserven liegen in der instabilen Region des Nahen und Mittleren Ostens. Im US-Senat ist derzeit ein Gesetzentwurf der Abgeordneten Lieberman und Warner anhängig, der eine CO2-Reduktion von 65 Prozent bis 2050 vorsieht und ein Cap\&Trade-System enthält. Auch wenn der Erfolg dieses Entwurfs unsicher ist, so wird das Thema spätestens 2009 unter der neuen Präsidentschaft und dem neuen Kongress wieder auf die Agenda kommen. Die demokratischen Präsidentschaftsbewerber, aber auch der Republikaner John McCain haben sich bereits für ein Cap\&Trade-System ausgesprochen.

Neben politischen und gesellschaftlichen Themen wird auch die Wirtschaft eine wichtige Säule der transatlantischen Beziehungen bleiben. Die Europäische Union und die USA sind die am engsten verflochtenen Wirtschaftsräume weltweit und für einander jeweils die mit Abstand wichtigsten Quellen für ausländische Direktinvestitionen. Fast 60 Prozent der amerikanischen Auslandsinvestitionen gingen 2006 in die EU. Europäische Firmen wiederum halten 75 Prozent am Gesamtbestand der ausländischen Direktinvestitionen in den USA. Beide Partner wickeln 40 Prozent des weltweiten Handels miteinander ab. Die USA und die EU tauschen täglich Waren im Wert von mehr als einer Milliarde Euro aus. Dank der transatlantischen Wirtschaftspartnerschaft wurden in den letzten Jahren in Europa und den USA rund 14 Millionen Arbeitsplätze geschaffen. Allein deutsche Unternehmen sichern 700.000 Arbeitsplätze in den Vereinigten Staaten. ${ }^{12}$ Beim EU-USA-Gipfel im April 2007 wurde eine Rahmenvereinbarung zur weiteren Vertiefung dieser Wirtschaftsintegration unterzeichnet. Mit Hilfe eines ,Transatlantischen Wirtschaftsrats' konnten Rechnungslegungsstandards und andere Regulierungen gegenseitig anerkannt werden - eine enorm wichtige Sache für die Unternehmen.

Eine Aufgabe, die noch vor uns liegt, ist die vernünftige und humane Gestaltung der Globalisierung. Bislang gehörten die USA und die EU-Staaten zu den größten Gewinnern der globalisierten Wirtschaft. Aber auch sie verspüren immer mehr den scharfen Wind des internationalen Wettbewerbs. Neue Wirtschaftsgroßmächte wie Indien und China, aber auch viele wirtschaftlich erfolgreiche und anpassungsfähige kleinere Staaten, schicken sich an, Marktanteile von uns zu erobern. Zugleich sind unsere Volkswirtschaften durch ihre internationale Vernetzung immer anfälliger für die ,Ansteckung' durch wirtschaftliche und finanzielle Schocks, die irgendwo auf der Welt ausgelöst werden. Die weltweiten Finanzturbulenzen durch die sogenannte, subprime loan crisis' und hohe Verluste von Bankinstituten in diesem und im vergangenen Jahr sind lehrreiche Beispiele. Auch Entwicklungen in der chinesischen Volkswirtschaft, bald die drittgrößte der Welt, werden immer größere Auswirkungen auf die wirtschaftliche Lage in Europa und den USA haben.

Wenn wir als Verbraucher immer günstiger einkaufen wollen, befördern wir zugleich die Verlagerung unserer Arbeitsplätze in Billiglohnländer mit niedrigen Umwelt- und Sozialstandards.

12 Siehe dazu z. B.: Daniel S. Hamilton, Joseph P. Quinlan: The Transatlantic Economy 2008, Executive Summary. Center for Transatlantic Relations, Washington D. C. 2008, unter: http://transatlantic.sais-jhu.edu/Publications/TransEcon2008_executive_summary. pdf.
Und weil wir als Anleger immer höhere Renditen erwarten, investieren Banken auch in bedenkliche, kaum abgesicherte Anlagenpakete und geraten anschließend ins Strudeln. Da die Gewerkschaften geschwächt worden sind, können sie häufig nur noch für einen privilegierten Kreis von Arbeitnehmern gute Einkommen aushandeln, während andere, tendenziell größer werdende Teile der Erwerbsbevölkerung von prekären und schlecht bezahlten Beschäftigungsverhältnissen leben. Diese wirtschaftlichen Entwicklungen haben politische Konsequenzen: Gesellschaften, in denen der soziale Friede gestört ist und die sich als schutzlos gegenüber fremden Bedrohungen empfinden, sind dafür anfällig, Werte von Demokratie und Liberalität in Frage zu stellen und ihr Heil bei autoritären und populistischen Politikern zu suchen. Patentrezepte gegen Probleme, die mit der im Prinzip positiv zu bewertenden Globalisierung einhergehen, werden sich nicht finden lassen. Aber wer, wenn nicht die beiden größten und am stärksten integrierten Wirtschaftsblöcke der Welt, soll sich um Lösungskonzepte für solche zentralen Fragen des 21. Jahrhunderts bemühen?

\section{Fazit}

Europa und die USA werden nach den Präsidentschaftswahlen vom November 2008 weiterhin eng zusammenarbeiten. Es bleibt ihnen auch gar nichts anderes übrig, um die drängenden Probleme unserer Zeit - asymmetrische Konflikte in Krisenregionen, die Verbreitung von Massenvernichtungswaffen, den Klimawandel, die Herausforderung der Globalisierung und viele andere - zu bewältigen. Für die Europäer bleiben die USA ohnehin der wichtigste Partner. Auch die amerikanischen Politiker wissen, dass die Schnittmenge an gemeinsamen Interessen und Werten mit keinem anderen Partner so groß ist wie mit Europa.

Nach dem Antritt der neuen Administration ist die Zeit günstig für eine noch engere transatlantische Kooperation. Die USA werden nach den schwierigen Erfahrungen im Irak versuchen, Verbündete für ihre Stabilisierungsbemühungen im Nahen Osten zu finden und könnten sich dabei zugänglicher für Argumente der Europäer zeigen, als es in den letzten Jahren manchmal der Fall war. Zugleich werden sie sich intensiver mit Themen wie Klimaschutz und Energiesicherheit beschäftigen, die schon jetzt weit oben auf der außenpolitischen Agenda der Europäer stehen. Die Europäer sollten in ihrem eigenen Interesse positiv auf amerikanische Angebote zur Zusammenarbeit eingehen. Wo immer Europa geeint auftritt, ist es zu einer wichtigen Stimme in der Weltpolitik geworden. Ohne und erst recht gegen die USA wird die EU ihre außenpolitischen Ziele jedoch nicht durchsetzen können.

Dabei werden Unterschiede in Meinungen und außenpolitischen Ansätzen zwischen Europa und den USA bestehen bleiben, gleichgültig, ob die nächste US-Regierung demokratisch oder republikanisch geführt wird. Beide Seiten sollten lernen, mit solchen Differenzen gelassen umzugehen, denn ein automatischer Gleichklang der Interessen wie im Kalten Krieg wird sich nicht mehr herstellen lassen. 